\title{
Le vote : son passé, son présent, son avenir
}

Vadja keel: minevik, olevik, tulevik

The Votian language: past, present, future

\section{Heinike Heinsoo}

Traducteur : Eva Toulouze

\section{(2) OpenEdition}

1 Journals

\section{Édition électronique}

URL : https://journals.openedition.org/efo/734

DOI : 10.4000/efo.734

ISSN : 2275-1947

Éditeur

INALCO

\section{Édition imprimée}

Date de publication : 1 décembre 2012

ISBN : 978-2-343-02592-6

ISSN : 0071-2051

\section{Référence électronique}

Heinike Heinsoo, «Le vote : son passé, son présent, son avenir », Études finno-ougriennes [En ligne], 44 I 2012, mis en ligne le 12 février 2014, consulté le 11 juillet 2021. URL : http://journals.openedition.org/ efo/734 ; DOl : https://doi.org/10.4000/efo.734

Ce document a été généré automatiquement le 11 juillet 2021.

\section{(c) (i) (8)}

Études finno-ougriennes est mis à disposition selon les termes de la Licence Creative Commons Attribution - Pas d'Utilisation Commerciale 4.0 International. 


\title{
Le vote : son passé, son présent, son avenir
}

\author{
Vadja keel: minevik, olevik, tulevik \\ The Votian language: past, present, future
}

Heinike Heinsoo

Traduction : Eva Toulouze

\section{Le vote est une langue en cours d'extinction}

1 Les «langues en danger " ont été subdivisées en trois groupes selon leur niveau de vitalité : les langues en danger, les langues en voie d'extinction, les langues éteintes. Les critères permettant de classer une langue dans une de ces catégories sont les suivants : 1. Les enfants ne la parlent plus; 2. elle n'est plus parlée que par quelques vieillards et 3. plus personne ne la parle. Il semblerait que la moitié des 6700 langues parlées dans le monde soient amenées à disparaître avant la fin du siècle. La seule chose qui soit susceptible ralentir ce processus, c'est l'initiative des gouvernements et des sociétés. Le programme Langues en danger de l'UNESCO (Endangered Languages Programme) essaye de faire le tour de la question et de l'évaluer. C'est sur la base du travail de l'UNESCO qu'a été mis en place l'atlas mondial des langues en danger (The Atlas of the World's Languages in Danger). Parmi les langues fenniques, on y trouve entre autres le vote et l'ingrien. Le vote n'est plus parlé par les enfants. Il n'y a plus que quatre ou cinq personnes âgées qui parlent couramment cette langue.

2 Traditionnellement, on distingue quatre dialectes du vote: le vote oriental, le vote occidental, le vote de Kukkus et celui de Kreevin. Ce dernier s'est éteint dans la première moitié du xIX $x^{e}$ siècle à proximité de Bauska, aujourd'hui en Lettonie. Dans les années 1960, il restait deux ou trois locuteurs du vote oriental, alors que le dialecte de Kukkus n'était parlé que par quelques individus dans les années 1970. Quant au dialecte occidental, il se subdivise à son tour en trois groupes : le groupe d'orko, celui de mätš, et celui de Vaipoole. 


\section{Le pays vote sur la carte}

3 En 1848, l'ethnographe Peter von Koeppen, venu de Saint-Pétersbourg, identifia dans le gouvernement de Saint-Pétersbourg un total de trente-sept villages votophones, habités par 5148 Votes. Il ne considérait pas les 150 habitants du village de Kukkus comme des Votes (Köppen 1967). En Ingrie occidentale, les villages votes étaient fort dispersés. Le village central d'Ingrie centrale parlant le dialecte d'ülägo était Kattila, et les principaux villages des zones lacustres du groupe mätš étaient Kõrvõttula, Mati, Savvokkala, ainsi que Jarvikoistšülä, qui a disparu pendant la guerre. En 1848, la population vote se répartissait par villages de la manière suivante :

- dans les villages occidentaux de la paroisse de Kattila, 162 Votes (9\%);

- dans les villages du groupe orko, 476 (27\%);

- dans les villages du groupe mätšs, 1152 (64\%);

-à Vaipoole, à l'époque de Peter von Köppeni, résidaient 632 Votes (plus de $10 \%$ de l'ensemble) (Köppen 1867).

Le vote disparut dans les villages des groupes orko et mätš dans les années 1960 ; dans les années 1990, même le souvenir de leur existence s'était estompé. Quand Hannu Hyttinen, un journaliste de Tampere, réalisa en 1997 un documentaire sur les Votes, «Le peuple des grand-mères " (en finnois : Mummojen kansa), au magasin du village de Mati plus personne n'était capable de répondre à la question КТО ТАКАЯ Водь? «Qui sont les Votes? » Une personne émit l'idée qu'il s'agissait peut-être d'une nationalité : « НАЦИОНАЛЬНОСТЬ МОЖЕТ БЫТЬ ${ }^{1}$ ». Еn 1960, Ariste observait que dans les groupes de villages orko ja mätš, le nombre de personnes parlant bien le vote était inférieur à vingtcinq ; à Vaipoole, il y en avait à peu près autant (Ariste 1960, p. 207).

Aujourd'hui la langue vote y a entièrement disparu, et même le souvenir qu'une langue autre que le russe ait été parlée dans la région a disparu. Dans les villages votes de la paroisse de Kattila, il y a un siècle et demi, les Votes étaient bien plus nombreux qu'à Vaipoole. Or dans cette dernière localité, le vote s'est préservé dans une certaine mesure. Ceci est dû, d'après Ariste, au fait que les Votes et les Ingriens de l'intérieur avaient migré dans les grandes villes, parmi les Russes, alors que les pêcheurs et navigateurs des villages de Vaipoole, au bord du golfe de Finlande, étaient davantage en contact avec des Ingriens, des Finnois et des Estoniens ; en raison de la parenté de ces langues entre elles, ils pouvaient utiliser, dans la communication, leur langue maternelle (Ariste 1981, p. 79).

6 Par rapport aux villages du groupe orko, Vaipoole se trouve au nord-ouest : aujourd'hui, Jõgõperä, Luuditsa et Liivtšülä sont les villages des derniers locuteurs du vote. Luuditsa et Liivtšülä ont été administrativement fusionnés en un seul village, qui porte le nom de Luuditsa; suite à l'incendie de 1912, le village de Rajo, où vivait la täätäjä Oudekki Figurova (1891-1978), qui a chanté à Paul Ariste beaucoup de chants (Ariste 1978, 1986, p. 30 et suiv.), est devenu un lieu de villégiature dont la population est russophone.

7 Il est difficile d'identifier ces villages sur la carte de l'oblast' de Saint-Pétersbourg, car leurs appellations officielles sont en russe : les noms ont été soit traduits soit adaptés au russe. Plus rarement, des villages ont un nom entièrement différent en russe, par exemple le principal village de Vaipoole, Jõgõperä, qui est en russe Krakolje (КРАКОЛЬЕ), ou encore Rajo, qui est en russe Mežnjaki (МЕЖНЯКИ). Le nom de Liivtšülä, 
en revanche, a été traduit en russe : il signifie "village des sables », et son nom russe, Peski (ПЕски), signifie «sable »; le nom Luuditsa, pour sa part, a été adapté : Lužicy (лужицы). Les noms des autres villages lives suivent ces mêmes modèles: Jarvikoištšülä, qui a disparu pendant la guerre, était en russe Babino (БАБино), Pontizõõ est Pontilovo, Poidelevo (понтилово, поидЕлЕво), Savvokkala est en russe Savikino (САвикино), le nom de Kattila a été traduit par Kotly (котлы) etc. Pour s'orienter dans le labyrinthe des noms, on peut faire appel à la carte pentalingue d'Ingrie (Inkeri. ингеРмАнлАндия. Ingermanland. Ingria. Ingeri - Inkeri 1992). Dans le cas de certains villages, chacun utilise le nom dans sa langue : par exemple le village au nom russe de Krakolje reste Jõgõperä pour les Votes, Joenperä pour les Finnois, et Joemperä pour les Ingriens.

Les langues votes en Ingrie occidentale

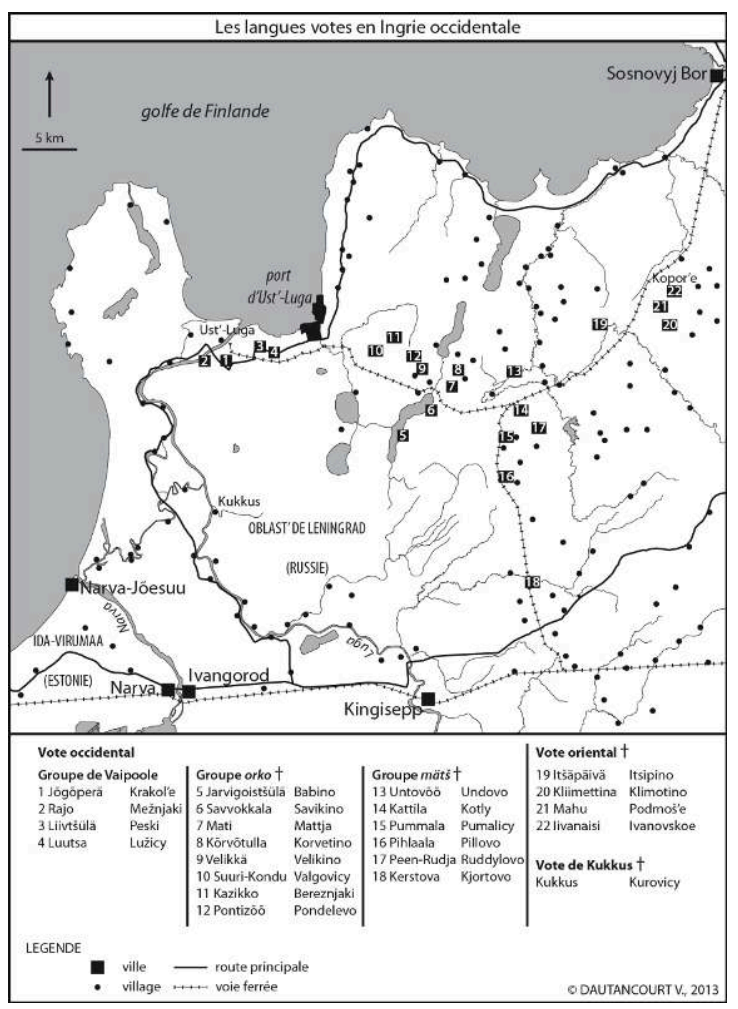

\section{Les locuteurs du vote}

\section{Le nombre des Votes au fil des temps : recensements et identité}

En 1848, le nombre des Votes est de 5 143. Il s'agit sans doute d'un recensement avant la lettre, et le calcul a dû être effectué assez sérieusement. Les chiffres suivants proviennent de différentes initiatives ou d'estimations approximatives des chercheurs (Köppen 1867).

1901 : 2 000-3 000 locuteurs du vote d'après Emil Nestor Setälä sur la base de ses travaux de terrain de 1889 (Suhonen, Posti 1964);

1915 : 1000 locuteurs du vote d'après Lauri Kettunen, après son troisième voyage en pays vote ; 

Deuxième Guerre mondiale, le nom « vote » ne figurait pas sur les passeports, mais il y avait par exemple ižor (Ижор) «ingrien» ou encore ingermanlandec/ingermanlandka (ИНГЕРМАНЛАНДЕЦ/ИНГЕРМАНЛАНДКА) «Finnois/Finnoise d'Ingrie ». En 2002, il devint possible de se déclarer vote : le recensement donna alors le chiffre de 73. Mais les habitants votophones des villages votes affirment que personne, à l'époque, n'était passé les recenser. Sur la base des données figurant sur les papiers d'identité, les recenseurs les ont manifestement considérés comme Russes. Le nombre réel de Votes, ou plutôt de locuteurs du vote, qui étaient en contact avec un environnement linguistique vote, était à l'époque sans doute d'une vingtaine. Avant le recensement d'octobre 2010, la journaliste Jana Romanova (de la revue Russkij reporter) a interrogé les derniers Votes sur leurs intentions au recensement (Romanova 2010). Voici des extraits de quelques réponses : Tatjana Naumova (née en 1941) : «Je vais bien sûr me déclarer vote, il ne peut pas être question d'autre chose »; Zina Saveljeva - « Bien sûr, vote, j'en ai assez d'avoir peur »; Aleksander Borissov (1932-2012) : «En fait, je m'en fiche »; Taissa Mihhailova (1934), Niina Lenivenko, Nikolai Nesterov : «Russe, comme sur mon passeport ». 


\section{Les locuteurs aujourd'hui}

21 Certains linguistes russes estiment que la plus jeune locutrice du vote est la fille d'un militant de Saint-Pétersbourg, qui a aujourd'hui la trentaine. Son père lui avait appris la langue quand elle avait cinq ans. Ni le père ni la fille n'ont leurs racines dans un village vote. Les derniers Votes sont des grands-parents, dont les enfants et les petitsenfants ne parlent pas vote. Cela fait vingt ans qu'il n'y a plus une seule famille où l'on parle vote. Les rares locuteurs du vote ne le parlent pas entre eux. La meilleure locutrice du vote de la dernière génération était Tatjana Prokopenko (1935-2003) : les influences des autres langues étaient peu sensibles dans son discours et elle pensait manifestement dans sa langue. Ses réflexions exprimées en live ont été sauvegardées par Madis Arukask, Taisto Raudalainen et Ergo-Hart Västrik dans leur film de 2004 « ... Et délivre-nous du mal ", où on la voit inventer des mots votes sans avoir recours au russe. Le décès de cette informatrice vitale et créatrice a réellement représenté la mort du vote vivant.

Les parents de Sergej Jefimov, né en 1954, sont d'origine vote, ils proviennent des villages de Liivtšülä et d'Ala-Luutsa. Jusqu'à ces derniers temps, Sergej vivait à Luuditsa. Ilmar Talve, un ethnographe estonien destiné à devenir professeur d'ethnographie à l'université de Turku, a photographié le 12 septembre 1942 Konstantin Jefimov (dit Kronni Nikon), grand-père de Sergej. Sergej avait commencé à perdre la maîtrise de la langue vote. C'est grâce à son épouse, Tatjana Jefimova, originaire des terres vepses et sans racines votes, qu'il a retrouvé la conscience de ses origines. Cela fait maintenant vingt ans que Tatjana œuvre en pays vote sans se ménager : elle a été pratiquement à l'origine de tout ce qui s'est fait autour du monde vote: le drapeau, les armoiries, l'hymne, les fêtes de village; elle anime le musée. Maintenant, Sergej travaille consciemment le vote ; avec son épouse, ils traduisent en russe les textes publiés par les chercheurs, inventent les noms votes des objets exposés dans le musée. En novembre 2011, ils ont téléchargé sur le site vote vatland.ru un texte en vote de Sergej Jefimov, "Promenade virtuelle à Luuditsa", où il commente d'anciennes photos.

23 Parmi les bons locuteurs, on trouve encore Zina Saveljeva, née en 1938 et résidant à Jõgõperä. Zina n'a assumé sa « votitude » que tardivement. Elle a dû surmonter la honte qu'elle éprouvait à parler le vote moins bien que sa tante Nadjoža Leonteva (1898-199?), qui avait grandi à Liivtšülä, et que le mari de celle-ci, Stjopa Leontev (1896-199?), qui ont été pendant des années les informateurs linguistiques de Paul Ariste. Zina a travaillé des années comme institutrice à l'école de Jõgõperä et son mari, sa fille et sa petite-fille sont fiers d'elle, même s'ils ne comprennent pas le vote. La maison des Saveljev se trouve à un endroit où il y avait antérieurement une école, qui a été transportée sur la côte de Lauga et transformée en club. Il est intéressant de noter que Zina est aussi capable d'écrire en vote. Elle le fait avec l'alphabet latin et son orthographe est aussi correcte que celle du dictionnaire vote. Zina Saveljeva a lu intégralement les épreuves de la première édition du dictionnaire des langues ouraliennes, et elle a fait des propositions de corrections (UKS 2004, 2011).

Niina Lenivenko est née en 1935 et elle a vécu toute sa vie à Jõgõperä, pour une grande partie avec sa fille Tanja, née en 1956, qui est handicapée et ne voit pas le vote d'un bon œil. Tania comprend en fait assez bien le vote, mais ne l'utilise jamais. Niina vit depuis une dizaine d'années dans le centre industriel d'Ust-Luga. Sa perception de sa propre 
identité est fluctuante, ainsi que ses représentations culturelles, qui sont très russes, car pendant des années elle a dirigé le travail du club et s'est consacrée à la diffusion de la culture russe. Nina Lenivenko est la dernière informatrice vote à avoir travaillé avec Paul Ariste. La dernière entrée du dossier Ethnologie vote $\mathrm{n}^{\circ} 3$ a été réalisée à Tartu, à l'hôpital de Maarjamõisa, où Ariste et Lenivenko étaient en même temps.

Paša Kartašova vit à Liivtšülä. Sa langue est très marquée par l'ingrien, et le russe a souvent tendance à s'y glisser. Mais elle parle sans complexes, et une année elle m'a raconté de nombreux contes en vote, par exemple «Maša et l'ours ", ainsi que "Les trois ours et le Petit chaperon rouge ». Elle s'en souvenait sans difficulté, car elle les avait racontés quelques années auparavant en russe à ses petits-enfants : en transposer le texte en vote ne lui a posé aucune difficulté.

Ivan Georgiev est né en 1928. Son père, Kiko Georgiev, raconta à Paul Ariste, il y a de cela plusieurs années, beaucoup d'histoires sur la vie de village et de savoureux récits de marins, qui sont parus dans différents recueils (par exemple Ariste 1977, 1982, p. 50-68). En 1980, le 27 juillet, le dernier jour de sa dernière expédition vote, Ariste notait : « Ce matin j'ai écrit et enregistré Kiko Georgiev » (VP, p. 184).

Ariste, Heinsoo, Markus et Rožanski ont noté soigneusement la langue des Votes de Vaipoole et en ont décrit les caractéristiques (Heinsoo 2002, 2006, Heinsoo, Kuusk 2004 ; Markus, Rožanski 2011). L'étude du vote a commencé par l'intérêt de quelques amateurs, a été portée par le travail sur le folklore et se retrouve désormais dans les notes de linguistes professionnels. Elle a été ainsi documentée et étudiée à fond (Heinsoo 2004 ; Heinsoo, Kuusk 2011).

\section{Situation sociale et politique}

En Ingrie Occidentale, comme partout en Russie, les kolkhozes sont un souvenir du passé et il est difficile de trouver du travail sur place. La construction du port d'UstLuga dans la presqu'île de Soikkola n'y a rien changé. Le port d'Ust-Luga a été développé pour contrecarrer les ports de la Baltique situés dans des États ne dépendant pas de la Russie (ports de Muuga en Estonie et de Riga en Lettonie) et s'en affranchir. Les installations du nouveau port sont censées voir passer vingt millions de tonnes de pétrole par an. D'autres terminaux sont en construction. Vladimir Poutine a promis qu'en 2018 Ust-Luga compterait parmi les dix principaux ports commerciaux au monde, déclarant : «Les perspectives d'Ust-Luga sont liées au développement d'une puissante industrialisation. Il faut construire des usines, des appartements et des infrastructures modernes. Cela veut dire que nous construisons une ville entièrement neuve pour 35000 à 40000 personnes ».

Tout ceci a une incidence sur le milieu de vie des populations riveraines. La construction du port nuit directement et indirectement aux villages de Vaipoole. Tous les terrains non construits ont été loués. Des milliers d'ouvriers du bâtiment boivent et jettent les bouteilles dans la forêt (la Russie est bien assez riche pour produire de nouvelles bouteilles, comme l'a déclaré un cadre moyen travaillant au port), la circulation entre les villages est aujourd'hui semblable à celle de l'artère principale d'une grande ville. En même temps, les chemins sont tellement abîmés par les poids lourds que les autobus ne peuvent guère les utiliser. Cette entreprise géante de construction a contribué à mobiliser la population pour la défense de ses intérêts. 
le 6 avril 2005 qu'a été créée l'Union pour la culture vote. Légalement, c'est une association à but non lucratif, dont les objectifs sont :

- l'étude et la préservation de la culture vote ;

- l'organisation de conférences, de séminaires, de colloques, d'expositions, de concerts, de festivals et de manifestations culturelles ;

- la participation à des activités d'édition et d'intervention dans les médias ;

- la préparation de matériaux d'information, d'outils didactiques et de programmes audiovisuels ;

- le développement et le maintien de contacts avec des organisations analogues;

- la protection et la préservation des monuments reconnus de la culture et de l'histoire votes.

L'association a rempli la plupart des objectifs qu'elle s'était fixés et continue à travailler activement.

Suite à l'action de l'Union pour la culture vote, le 13 octobre 2008, les Votes ont été ajoutés à la liste des petits peuples autochtones de la Fédération de Russie ${ }^{2}$. Cette liste est composée des peuples autochtones de Russie dont les effectifs sont de moins de 50000 personnes et qui mènent sur leur territoire d'origine un mode de vie traditionnel. Être sur la liste garantit un certain nombre de droits, mais les organes administratifs n'ont que le «droit» (et pas le devoir) de soutenir le mode de vie des petits peuples et d'aider à préserver leur culture.

Le 31 novembre 2008, le journal local commenta l'événement en ces termes : «Ce petit peuple de 73 personnes, au prix de grands efforts, a maintenu ses traditions et sa langue, publie des manuels et leurs enfants ont des cours de langue (certes, actuellement, non obligatoires)» (31.10.2008). On identifie ici des accents propagandistes: les grands distribuent les éloges et n'oublient pas totalement les petits. Or aucun manuel n'est paru et aucun cours de vote n'existe, car il n'y a pas à l'école d'enfants de parents votophones. Et il ne peut pas y en avoir, car les enfants de parents votophones sont adultes et n'ont jamais entendu leurs parents parler vote. De plus, ils ne vivent même pas dans la région. En termes de classe d'âge, ce sont leurs enfants qui pourraient aller à l'école de Krakolje, mais ceux-ci, dans le meilleur des cas, y vont l'été voir leurs grands-parents.

Dans le nouveau passeport russe, la nationalité n'est pas mentionnée et le mode de vie traditionnel est depuis longtemps devenu impossible, du fait de l'époque et de la construction du port d'Ust-Luga.

Le port subventionne la construction d'un musée, qui a été achevé fin 2012. Le bâtiment, une belle maison en rondins, est la copie exacte de la maison des Borissov à Jõgõperä. Maria Petrova (née Borissova), qui a vécu jusqu'à sa mort dans la ferme de ses parents, y accueillait encore dans les années 1990 ses deux sœurs et son frère, qui vivaient dans leur village; ils échangeaient des nouvelles exclusivement en vote. C'est le frère, Ol'o Borissov, qui est décédé en dernier, en janvier 2012. À l'heure actuelle, c'est Vera Safronova, fille de Maria, qui préserve leur mémoire et a ouvert dans son village un musée en plein air. Les écoliers de Jõgõperä, les touristes, les linguistes, tous peuvent aller voir les objets qui se sont accumulés dans la ferme pendant plus d'un siècle.

Le musée de Luuditsa est prêt, mais aucune ressource financière n'est prévue pour son entretien. 
37 Sur la colline en face de l'école de Jõgõperä, les coupoles dorées de l'église en construction s'élèvent vers le ciel. La basilique est particulièrement grandiose, ses alentours sont étonnamment propres, les routes et les lampadaires ressemblent davantage à ceux d'une riche station balnéaire qu'à ceux d'un village où l'école va bientôt fermer. C'est là aussi un corollaire de la construction du port, comme il est de coutume en Russie : qui commet un péché construit une église. L'école, elle, va fermer parce que dans les villages il n'y a pas d'enfants. Les enfants étaient amenés en bus du centre industriel d'Ust-Luga jusqu'à l'école-lycée construite en 1968. L'école deviendra un hôtel pour les constructeurs et les travailleurs du port, qui ont de plus en plus besoin de logements. Or elle fonctionnait dans ce village depuis la fin du XIX $\mathrm{X}^{\mathrm{e}}$ siècle.

\section{Les activités culturelles}

Si les Votes de Vaipoole ont préservé leur identité, c'est indubitablement grâce aux actions que les militants votes ont engagées dès la fin du siècle dernier.

\section{Le musée}

Le premier musée vote, fondé en 1997 dans la ferme des parents de Sergej Jefimov, brûla en 2001. Avant l'incendie, il contenait soixante-dix objets ethnographiques et plus de deux cents anciennes photos (Ernits 2006, p. 83). À l'aide de leurs amis, les Jefimov se sont construit une nouvelle maison, mais ils n'ont plus voulu de musée sous leur toit. En 2005, le musée alla s'installer dans une maison proche de la leur, et il fut ouvert aux visiteurs lors des festivités du village de Luuditsa en 2005. Ce nouveau musée brûla lui aussi à l'automne 2006 et il ne fut possible de sauver que peu de choses. À l'heure actuelle, les Jefimov animent activement un musée sur Internet, watland.ru. Quel avenir aura le musée dont la construction s'est achevée en 2012, nul ne le sait.

\section{La fête du village de Luuditsa}

40 C'est en 2000 que l'anniversaire du village de Luuditsa, le "Potluck de Luuditsa », a été fêté pour la première fois.

41 Les informations trouvées dans le musée ethnographique de Saint-Pétersbourg permettaient de supposer que le village, en 2000, allait avoir cinq cents ans. Tatiana Jefimova prit l'initiative. Depuis, l'anniversaire du village est fêté tous les ans au mois de juillet. Le programme est très riche: chants, danses, banquet. Il rassemble la population du village et les amis venus de près et de loin. En décembre 2001, Aleksandr Gurinov, un artiste de Saint-Pétersbourg, a mis au point un drapeau vote, qui est levé solennellement avec celui de la Fédération de Russie. Un autre artiste pétersbourgeois, Vladimir Zernov, mieux connu comme poète russophone d'Ingrie, conçut quelque temps plus tard des armoiries votes. L'hymne a été inspiré par un chant chanté en russe par Fenja Petrova, une Ingrienne habitant Luuditsa - «Pardonne-moi, mon chéri, adieu $^{3}$ ", qui était chanté à Luuditsa en accompagnement du défilé nuptial vers l'église. C'est Ivan, le fils de Tatjana Jefimova, qui en a fait l'hymne vote. 


\section{Le lycée de Krakolje}

42 L'école de Jõgõperä, après la perestroïka, a été fort active dans la promotion de la langue et de la culture des Votes. Leur groupe musical d'enfants, dont la composition se renouvelle, a chanté pendant des années des chants votes; c'est dans ce lycée que le linguiste Mehmet Muslimov, qui a soutenu sa thèse de doctorat sur les contacts linguistiques en Ingrie occidentale, enseigne le vote depuis les années 1990. Muslimov a d'ailleurs mis sur internet cinq cours de vote (http://www.vatland.ru/). Il a été assisté dans son travail d'enseignant par les Votes Zinaida Saveljeva et Tatjana Prokopenko (1935-2006), avec parfois la participation de Tatjana Jefimova. En tout cas, leurs cours ont permis au groupe musical de jeunes «Linnut » («Pääskolinnut ») d'apprendre plus facilement les chants et de petits sketches en vote. Depuis le nouveau millénaire, l'enseignement a eu lieu de manière moins systématique. En 2011-2012, le vote était enseigné par Nikita Djačkov, un habitant de Luuditsa d'origine ingrienne. Il est né en 1987, a fait ses études universitaires à Saint-Pétersbourg et a travaillé au musée de Vistino. On espère que lorsqu'il aura terminé son service militaire, il retournera travailler au nouveau musée vote. Nikita est l'un des militants formés par Muslimov à Saint-Pétersbourg.

\section{Bestiarum et les expositions de photos de Jekaterina Kuznetsova}

Le vote est la langue dans laquelle chantent les jeunes folkloristes de Saint-Pétersbourg qui ont formé le groupe Bestiarum, avec l'artiste Jekaterina Kuznetsova, qui y chante et y joue divers instruments. Ils ont une musique vote profondément liée à ses racines, en même temps moderne et populaire ${ }^{4}$. De plus, de nombreuses expositions de photos sur le pays vote ont été présentées à Saint-Pétersbourg, et on les trouve sur ce même site.

\section{La langue - hier et aujourd'hui}

Lauri Kettunen, en 1911, lors de son premier voyage en pays vote, pensait que le dialecte de Jõgõperä était un mélange de vote, d'ingrien et d'estonien (1945, p. 204).

En 1925, Dmitri Tsvetkov, étudiant d'origine vote de l'université de Tartu, écrivait dans la revue La langue estonienne ${ }^{5}$ :

«Pour les Votes, la langue de la religion est tout à fait étrangère. Il y a soixante ou quatre-vingts ans, seuls $10 \%$ des Votes comprenaient le slavon et la moitié d'entre eux le russe... Ces derniers temps, dans toutes les branches, l'assimilation s'accélère. Un exemple: dans une maison vote, où on parle vote, arrive une belle-fille ingrienne. Que se passe-t-il ? Tous les membres de la famille, même les personnes âgées, se mettront à parler l'ingrien » (Tsvetkov 1925, p. 42 -43).

Ilmari Talve séjourna en 1942, entre les 11 et 13 septembre, à Vaipoole; il écrivit à propos de la langue : "Le vote est parlé, mais j'ai l'impression que ce n'est plus une langue vivante, l'ingrien et le finnois sont davantage utilisés. Entre eux, les Votes parlent une langue dans laquelle l'influence ingrienne est considérable » (Talve 1990, p. 63-64). Dans les années 1950, Paul Ariste caractérisa la langue de Vaipoole comme une langue mixte; dans les villages votes, disait-il, on pouvait trouver des gens qui croyaient parler couramment le vote, mais qui ne parlaient qu'un mélange de vote, d'ingrien et de finnois (Ariste 1960, p. 203-204). En 1967, Ariste écrivait dans son journal 
qu'à Luuditsa et à Liivtšülä, dans chaque maison on parlait une langue fennique : vote, vote métissé d'ingrien ou ingrien métissé de vote (Ariste VP 2005, p. 97).

\section{Éducation, langue et langue littéraire}

47 Depuis plusieurs générations, les Votes parlent également, en plus du vote, l'ingrien et le russe. L'unique intellectuel vote, Dmitri Tsvetkov, né le 30 août 1890 à Jõgõperä et mort le 26 août 1930 à Narva, a fait l'objet d'un livre détaillé et passionnant par Enn Ernits (Ernits 2009). Lauri Kettunen, professeur à Tartu, avait invité Tsvetkov à y faire ses études. C'est à l'aide d'une bourse de la Société de la langue estonienne ${ }^{6}$ qu'il put achever le manuscrit d'un dictionnaire vote-estonien (Tsvetkov 1995), alors qu'il avait déjà mis au point une grammaire (Tsvetkov 2008). Tsvetkov, toutefois, ne devint pas votologue, mais slaviste, et il enseigna à l'école d'Alajõe. Aujourd'hui, sa grammaire n'est pas utilisable dans l'enseignement, mais son dictionnaire présente une collection de mots tout à fait précieuse (Kettunen, Posti 1932, p.118-144). En 1925, Tsvetkov porta un jugement sur le vote :

«Les Votes ne font pas la différence entre Ingriens et Votes. Les jeunes filles votes, qui sont bonnes chanteuses, chantent toujours ce refrain, censé parler d'elles : “BCE ИЖОРКИ КРАСВЕНКИЕ” (Toutes les Ingriennes sont belles) - paroles provenant d'un chant populaire russe» (Tsvetkov 1925, p. 43).

Tsvetkov commente là la confusion identitaire. La même confusion se retrouve plus tard dans la bouche d'autres informateurs. Dunja Mihailova, une bonne informatrice, nièce de Dmitri Tsvetkov, dit : «À quelques rares exceptions près, les Votes ont suivi l'école uniquement en russe (en 1936-1937, les enfants votes suivaient le programme à l'école de Luuditsa en ingrien)». Dmitri Tsvetkov, en 1925, écrivait: «Tout en condamnant de manière absolue la politique de l'empire russe à l'égard des terres à peuplement autochtone, je dois constater que la fondation d'écoles russes en milieu vote n'a suscité chez ces derniers aucune réaction hostile » (Tsvetkov 1925, p. 42-42).

Les Votes n'ont jamais eu de langue littéraire.

En 1874, Kieletär publia des traductions du « Notre Père » dans les langues fenniques (Ahlqvist 1874). La traduction vote se présente ainsi :

Izä med'ee kumpa ôlet taivaiza, pühätü ôlkoo nimes sinun, litši tulkoo sinu valtas, õlkoo sinun tahtoos nii maaza kui taivaiza; anna meid'ee tänänne ôkaõivädne leipäni ja anna meile med'deevôlkani anteehsi kui i müö anteehsi annamma med'd'dee võlgaliizile ja älä saata meitä manituhseesee a päässä meitä pahassa ku sinun on valta $i$ võima i slaava igää kõikkee. (Ahlqvist 1874, p.20).

51 On peut considérer que ce texte est la première traduction, mais son auteur reste inconnu.

Actuellement on travaille sur les questions d'orthographe. Les grammaires publiées reposent sur la langue du village occidental de Kattila (mätši) (Ahlqvist 1856, Ariste 1948, 1968). Mais celui-ci ne convient pas comme base d'une langue littéraire moderne. Le dialecte de Jõgõperä, avec des formes fortement réduites, est particulier et s'est rapproché à plusieurs égards de l'estonien. Les trois villages de Vaipoole présentent d'importantes différences entre eux, qui concernent surtout les voyelles finales. L'ouvrage de E. Markus et de F. Rožanski paru en 2011 «La langue vote contemporaine $^{7}$ » rend compte de manière détaillée de la langue vote parlée 
aujourd'hui, c'est à dire des dialectes de Jõgõperä et Liivtšülä-Luuditsa (Markus, Rožanski 2011). phonème tš $(\check{c})$, qui est depuis longtemps le trait le plus caractéristique du vote, par le graphème $c$. Sans doute, le problème tenait à la difficulté de noter ce phonème, et l'argument présenté en faveur de l'utilisation du $c$ est relativement simple : ceux qui en ont besoin sauront bien de quel phonème il est question. L'utilisation de la voyelle finnoise $y$ introduit un élément d'hétérogénéité dans le système vocalique, puisque celui-ci contient le graphème ó, caractéristique de l'estonien (Kuznetsova 2010, Jefimova 2010). Le dictionnaire du vote en sept volumes ${ }^{9}$ utilise une notation simplifiée reposant sur l'alphabet estonien (1990-2011). Or les décalages entre cet immense dictionnaire, qui rend compte de tous les dialectes, et le mode d'écriture adopté localement ne peuvent que gêner l'utilisation du dictionnaire. Maintenant, le dernier alphabet rendu public sur le site ressemble à celui du dictionnaire vote (cf. http:// vatland.ru./lang/index.php). 


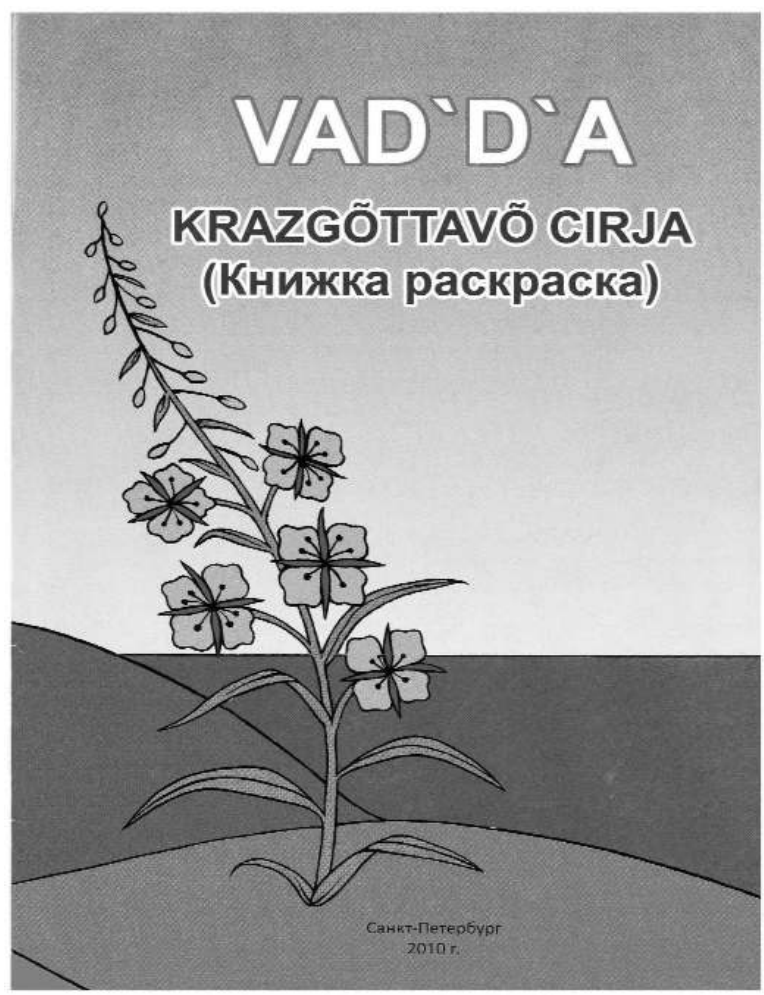

À la fin, on trouve l'alphabet, avec les sibilantes palatalisées, les $r^{\prime}$ et $t$ ', l'affriquée $\check{c}$ / tš) notée par $c$ et $c$ par ts. Dans ce livre, une hirondelle traverse les dix-neuf pages, et ce qu'elle voit est décrit en phrases simples. Sur la première page, on trouve encore trois phrases introductives: Vad'd'amaa on maa Soomõ Lahõ lõunad rannõz. Se on kõvi lusti. Kassõnn ammuzôlt aigalt eletä vad'd'alaizõd', qui veulent dire : « Le pays vote se trouve sur la côte sud du Golfe de Finlande. Il est très beau. Ici, depuis des temps anciens, vivent les Votes ».

Tatjana Jefimova a édité aussi un calendrier vote, Vad'd'a päivüzikko 2011 (Jefimova 2010). Ce calendrier correspond au Calendrier populaire vote de Paul Ariste en russe et en vote (Ariste 1969).

Le correcteur de ces deux éditions est Mehmet Muslimov. Le calendrier contient des néologismes. Le texte original est pour la plupart du dialecte de Liivtšülä-Luuditsa, mais on y trouve aussi les chutes finales qui caractérisent le parler de Jõgõperä. Les citations du calendrier d'Ariste sont présentées sous leur forme originale.

61 Enfin, en 2012 a été publié un aperçu historico-ethnographique du costume populaire vote La poupée vote en papier ${ }^{11}$ (Kuznetsova 2012), dont l'auteur est Jekaterina Kuznetsova. Le livre se présente comme une poupée en papier à colorier par les enfants, et il rend compte des évolutions du costume vote féminin au cours des siècles. Les rares commentaires sont en russe.

\section{Les problèmes de la lecture et de l'écriture du vote dans la pratique}

Pendant l'été 2010, 2011 et 2012, Heinike Heinsoo a organisé à Jõgõperä des cours de vote de quatre jours appelés "Des petits enfants à la grand-mère ${ }^{12}$ ". Il y avait une vingtaine de participants. On y trouvait plus de grand-mères que de petits enfants, ceux-ci étaient peu nombreux, et peu actifs. En 2012, les enfants n'étaient associés 
qu'un seul jour, pour apprendre des chansons. Aune Kämäräinen a commenté ces cours dans la revue Inkerin Kulttuurikanava (Kämäräinen 2010, 2011, 2012)

Dans ces cours, le problème de la lecture et de l'écriture a émergé de manière aigüe. Lors de la première édition, en 2010, l'enseignement était trop classique, il reposait largement sur les textes écrits et les apprenants devaient eux aussi beaucoup écrire. Les deux exercices posaient problème. En revanche, la lecture et le chant de textes de chants populaires marchèrent étonnamment bien, de même que la lecture de textes courts, de proverbes et de devinettes. L'année suivante, en 2011, les exercices d'écriture furent réduits au minimum, dans la mesure où les outils existaient sous forme imprimée. L'apprentissage était favorisé par une forte dimension ludique des cubes-morphèmes, des cartes de couleur que les personnes devaient deviner, des devinettes coupées en séquences, des fiches lexicales déjà prêtes... Mais seule Zina Saveljeva, le jeune employé du musée d'histoire locale de Soikkola d'origine ingrienne Nikita Djačkov et dans une moindre mesure Nina Vittonk (la sœur de Sergei Jefimov) se montrèrent en mesure de noter eux-mêmes des textes en vote. Les textes utilisés étaient systématiquement écrits comme le dictionnaire et les résultats sont patents, puisque le nouvel alphabet est désormais utilisé dans le site internet. Ainsi, après un accouchement très douloureux, la langue littéraire vote en est arrivée au point où elle est identique à celle utilisée dans le dictionnaire de 1990. La roue a été réinventée..

Ainsi, une langue littéraire prend forme, mais sera-t-elle utilisée par les Votes euxmêmes? On peut en douter. Il est pourtant bon de voir que la lecture de textes écrits tout prêts fonctionne assez bien. Pour que la langue littéraire puisse s'enraciner, il serait utile que paraisse un dictionnaire russe-vote. Celui-ci aiderait aussi à l'apparition d'une production originale. Et il serait également temps d'établir un livre de lecture pour débutants. Et de décider de son contenu.

Il est inutile de discuter du caractère juste ou pas juste de cette langue, il faut trouver un compromis entre les derniers locuteurs de deux villages. Il en a été ainsi pour la création du finnois et de l'estonien, mais le processus lui-même et la stabilisation ont pris des siècles. Les derniers locuteurs du vote ont moins de temps à leur disposition.

Le modèle de Haugen (Haugen 1953), modèle classique des mutations linguistiques, illustre le processus de la manière suivante :

- $\mathrm{A}>\mathrm{Ab}>\mathrm{AB}>\mathrm{aB}>\mathrm{B}$

- $\mathrm{A}=$ milieu de communication unilingue,

- $\mathrm{Ab}=$ bilinguisme avec prédominance de $\mathrm{A}$,

- $\mathrm{AB}$ = bilinguisme équilibré

- $\mathrm{aB}=$ bilinguisme avec prédominance de $\mathrm{B}$

- $\mathrm{B}=\mathrm{B}$ est l'unique langue de communication.

Le vote en est arrivé à une étape où, en milieu naturel, il est devenu un ancien souvenir d'enfance. Deux ou trois locuteurs seulement en sont à l'étape $\mathrm{aB}$, les autres sont entièrement passés à B, c'est à dire au russe. Mais depuis l'automne 2012 il existe une page Facebook présentant le vote, qui enseigne à qui veut les apprendre des mots en vote (http://www.facebook.com/VaddaTseeliVatjaaSanaPaivassa). Il y a déjà plus de cent apprenants... 


\section{BIBLIOGRAPHIE}

\section{Principales œuvres sur la langue et la culture votes}

\section{Films}

Väinölän lapset. Vatjalaiset. Helsingin yliopisto tiedottaa (Les enfants de Väinola. Les Votes), 14 mn, 1996.

Mummojen kansa (Le peuple des grand-mères), Hannu HYTTINEN, 27 mn, 1997.

Akadeemik Ariste kaks armastust. Vadjalased (Les deux amours de l'académicien Ariste. Les Votes), 22 mn, Madis ARUKASK, Ergo-Hart VÄSTRIK, Taisto RAUDALAINEN, ETV 2001.

Ja päästa meid ära kurjast (Et délivre-nous du mal), 60 mn, Taisto RAUDALAINEN, Ergo-Hart VÄSTRIK, ETV 2004.

ВОДЬ. ПРОШЛОЕ И НАСТОЯЩЕЕ ИЗЧЕЗЮЩЕГО НАРОДА (Les Votes. Le passé et le présent d'un peuple en voie de disparition), Olga KONKOVA, киностУдия „этнос“, 2003.

\section{Monographies}

Târnăveni KETTUNEN Lauri, 1915, Vatjan kielen äännehistoria. (Erikoispainos aikakauskirjasta Suomi), Helsinki.

KETTUNEN Lauri, 1930, Vatjan kielen äännehistoria, Suomalaisen Kirjallisuuden Seuran Toimituksia 185.osa, Helsinki.

RäNK Gustav, 1960, Vatjalaiset, Suomalaisen Kirjallisuuden Seuran Toimituksia 267, Helsinki.

TALVE Ilmari, 1981. Vatjalaista kansankulttuuria. Helsinki: Suomalais-Ugrilainen Seura.

\section{Grammaires}

AHLQVIST August, 1855, Wotisk grammatik jemte språkprof och ordförtrckning. Acta Societatis Scientiarum Fennicæ. Tomi qvinti. Helsingforsiæ.

ARISTE Paul, 1948, Vadja keele grammatika, Nõukogude Soome-ugri Teadused 9, RK „Teaduslik Kirjandus", Tartu.

ARISTE Paul, 1968, A grammar of the Votic Language, Indiana University Publications, Uralic and Altaic Series, volume 68.

Markus, Rožanski 2011= МАРКУС Е.Б., РОЖАНСКИЙ И. 2011. СОВРЕМЕННЫЙ ВОДСКИЙ ЯЗЫК. ТЕКСТЫ И ГРАММАТИЧЕСКИЙ ОЧЕРК. ТОМ І.,ІІ. САНКТ-ПЕТЕРБУРГ, НЕСТОР-ИСТОРИЯ

TŠERNJAVSKI Vitali, 2005. “VaĎĎa tšeeli (Izeõpõttaja)” (venäjänkielinen, sisältää vatja-venäjä ja venäjä-vatja sanakirjan, n 2000 sanaa).

TSVEткоv Dmitri, 2008, Vadja keele grammatika, Eesti keele Sihtasutus. Manuscrit: ЭСИМЕЙН ВАДДЯ чэЭЛЭ ГРАМААТИКК. СОСТ. Д.ЦВЕТКОВ 1922.

\section{Dictionnaires}

KeTTUNEN Lauri, 1986, Vatjan kielen Mahun murteen sanasto; Toim. Jarmo Elomaa, Eino Koponen ja Leena Silferberg, Castrenianumin toimitteita 27, Helsinki.

LAAKSO Johanna, 1990, Vatjan kielen käänteissanasto, Lexica Societatis fenno-ugricae XXII, Helsinki 1989. 
POSTI Lauri, SUHONEN Seppo, 1980, Vatjan kielen Kukkosin murteen sanakirja, Kotimaisten kielten tutkimuskeskuksen julkaisuja 8, Helsinki.

Vadja keele sõnaraamat. 1. 1990 (A-J), 2. 1994 (K), 3. 1996 (L-M), 4. (N-R) 2004, 5. (R-S) 2006, 6. (̌̌- T)

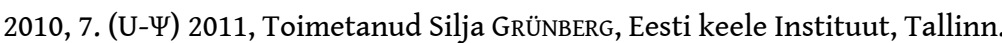

TSVETKOV Dmitri, 1995, Vatjan kielen Joenperän murteen sanasto, Toim. Johanna LAAKSO, Lexica Societatis Fenno-ugricae XXV, Helsinki.

Thèses

HEINSOO Heinike, 1987, ВЗАИМООТНОШЕНИЯ СКАЗУЕМОГО И ПОДЛЕЖАЩЕГО В ВОДСКОМ ЯЗЫКЕ, Tartu ( $M \bar{a}$ ja pūd lēväd, meid eb lē. The Subject and the predicate in Votic. Linguistica Uralica. Supplementary series / Volume 4. Teaduste Akadeemia Kirjastus, Tallinn 2010)

LAUERMA Petri, 1993, Vatjan vokaalisointu, SUST 214, SUS, Helsinki.

МАРКУС Е. Б., 2006, ТИПОЛОГИЯ МОРФЕМНОГО ВАРИРОВАНИЯ (НА МАТЕРИАЛЕ МОРФОНОЛОГИЧЕСКИХ СИСТЕМ ГОВОРОВ ВОДСКОГО ЯЗЫКА. ИСТИТУТ ЯЗЫКОЗНАНИЯ РАН МОсКВА.

WINKLER Eberhard, 1997, Krewinisch. Zur Erschließung einer ausgestorbenen ostseefinnischen Sprachform, Veröffentlichungen der Societas Uralo-Altaica, Band 49, Harrassowitz Verlag.

VÄSTRIK Ergo-Hart 2007, Vadjalaste ja isurite usundi kirjeldamine keskajast 20.sajandi esimese pooleni. Artiklitekstid, representatsioonod ja tõlgendused. Dissertationes Folkloristicae Universitatis Tartuensis 9, Tartu.

\section{Bibliographie}

AHLQVIST August, 1855, Wotisk grammatik jemte språkprof och ordförtrckning, Acta Societatis Scientiarum Fennicæ, Tomi qvinti, Helsingforsiæ.

AHLQVIST August, 1874, "Isämeidän-rukous länsisuomalaisilla kielillä", Kieletär I : 2, Helsinki, 19-23.

ARISTE Paul, 1948, Vadja keele grammatika, Nõukogude Soome-ugri Teadused 9, Tartu, RK „Teaduslik Kirjandus“.

ARISTE Paul, 1957, "Vatjalaisten nykyisyydestä", Virittäjä, 119-123.

ARISTE Paul, 1960, „Tänapäeva vadjalastest“, EMA XVII (1960), 203-224.

ARISTE Paul, 1968, A grammar of the Votic Language, Indiana University Publications, Uralic and Altaic Series, volume 68, Bloomington, Indiana University.

ARISTE Paul, 1969, Vadja rahvakalender, Tallin, Eesti Teaduste Akadeemia.

ARISTE Paul, 1977, Vadja muistendid, Tallinn, Valgus.

ARISTE Paul, 1978, „Oudekki Figurova“, Keel ja kirjandus 6, 348-350.

ARISTE Paul, 1981, Keelekontaktid. Eesti keele kontakte teiste keeltega (Eesti NSV Teaduste Akadeemia Emakeele Seltsi Toimetised nr 14), Tallinn, Valgus.

ARISTE Paul, 1982, Vadja pajatusi, ENSV TA ESA Toimetised 18, Tallinn, Valgus.

ARISTE Paul, 1986, Vadja rahvalaulud ja nende keel, Eesti NSV Teaduste Akadeemia Emakeele Seltsi Toimetised nr.22, Tallinn, Valgus.

ERNITS Enn, 2006, «ОБ ОБОЗНАЧЕНИИ ЗВУКОВ В ВОДСКОМ ЯЗЫКЕ», Linguistica Uralica 1, 1-9. 
ERNITS Enn, 2006a, Vadja keel ja kultuur ajalookatlas, Õpetatud Eesti Seltsi aastaraamat 2004-2005, Tartu, 2006.

ERNITS Enn, 2010, Vadja kirjaviisist ja sõnaloomest, ESUKA - JEFUL 2010, 1 - 1, 41-56

ERNITS Enn, 2010a, Väärt lugemisvara vadja muistendite ja muinasjuttude näol, Keel ja Kirjandus, 857-860.

ERNITS Enn, 2009, Vadja haritlane Dmitri Tsvetkov. Tartu, Eesti Kirjandusmuuseumi Teaduskirjastus.

HAUGEN Einar, 1956, Bilingualism in the Americas. A bibliography and research guide, Montgomery, University of Alabama Press.

HEINSOO Heinike, 2002, Jõgõperä vadjalaste keelekasutus, Fenno-Ugristica 24, 95-119.

HEINSOO Heinike, 2006, Paul Ariste ja Vaipoole vadjalased, Emakeele Seltsi Aastaraamat 51 (2005), 222-238.

HEINSOO Heinike, 2004, "Investigating obsolence: Votic", Fenno-Ugristica 26, 10-16.

HEINsoo Heinike, KuUSK Margit, 2004, No ku tulõb unõhtamizõ kõrt, ni õma tšeeli va jääb, Uralistika mü̈̈did ja faktid, Fenno-Ugristica, 17-36.

HEINSOO Heinike, KUUSK Margit, 2011, “Neo-renaissance and revitalization of Votic - who cares?” ESUKA - JEFUL 2 - 1, 171-184

INKERI 1992, Inkeri, ИНГЕРМАНЛАНДИЯ, Ingermanland, Ingria, Ingeri, (Tiekartta, КАРТА ДОРОГ, Vägkarta, Straßenkarte, Road map, Teede kaart), toim. Roland RANDEFELT, Ingriainfo OY.

Jefimova 2011 = ЕФИМОВА ТАТЬЯНА, Vad'd'a päivüzikko 2011, АНО оБЩЕСТВо водской кУльТУРЫ, САНКТ-ПЕТЕРБУРГ.

KeTtUNEN Lauri, Posti Lauri, 1932, Näytteitä vatjan kielestä, Suomalais-Ugrilaisen Seuran Toimituksia LXVIII, Helsinki.

KeTTUNEN Lauri, 1945, Tieteen matkamiehena. Kaksitoista ensimmaista retkeä 1907-1918, PorvooHelsinki, Söderström.

KöPPEN Peter von, 1867, Erklärender Text zu der ethnographischen Karte des St. Peterburger Gouvernements, St. Petersburg.

Konkova 2009 = КОНЬКОВА О.И., ПРЕДАНИЯ И СКАЗКИ ВОДСКОГО НАРОДА. Vad'd'aa rahvaa jutud ja kaazgad, СОСТАВИТЕЛЬ И АВТОР ВСТУПИТЕЛЬНОГО РАЗДЕЛА О.И.КОНЬКОВА, САНКТ-ПЕТЕРБУРГ: МУЗЕЙ АНТРОПОЛОГИИ И ЭТНОГРАФИИ ИМ. ПЕТРА ВЕЛИКОГО (КУНСТКАМЕРА), ЏЕНТР КОРЕННЫХ НАРОДОВ ЛЕНИНГРАДСКОЙ ОБЛАСТИ.

KUZNETSOVA Ekaterina, MUSLIMOV Mehmet, 2010, Vad'd'a. Krazgõttavõ cirja (КНИЖКА РАСКРАСКА), ХУДОЖНИК Е. КУЗНЕЦОВА, ТЕКСТ М. МУСЛИМОВ, Е. КУЗНЕЦОВА, САНКТ-ПЕТЕРБУРГ.

KUZNETSOVA Ekaterina, 2012, Vad'd'a paperi-lello, ВОДСКАЯ БУМАЖНАЯ КУКЛА, НАРОДНЫЙ ВОДСКИЙ ЖЕНСКИЙ КОСТЮМ ХІІІ -НАЧ. ХХ ВЕКОВ, ХУДОЖНИК ЕКАТЕРИНА КУЗНЕЦОВА, САНКТ- ПЕТЕРБУРГ, ОБЩЕСТВО ВОдской кЛЬТУРЫ.

KÄMÄRÄINEN Aune, 2010, Vatjan kulttuuri heräilee, Inkerin Kulttuurikanava. Inkerin kulttuuriseuran jäsenlehti 2/2010, 4-6.

KÄMÄRÄINEN Aune, 2011, Uudet konstit vauhdittavat vatjan kurssia, Inkerin Kulttuurikanava Inkerin kulttuuriseuran jäsenlehti, 2/2011, 4-5. 
KÄMÄRÄINEN Aune, 2012, Vatjan kesäkurssi - kesän kohokohta. Vatjan kielen ja kulttuurin kurssi Krakoljen koululla jo kolmatta kertaa, Inkerin Kulttuurikanava. Inkerin kulttuuriseuran jäsenlehti 1/2012, 12-13.

Lensu 1930 = ЛЕНСУ, Я.Я, «МАТЕРАЛЫ ПО ГОВОРАМ ВОДИ», ЗАПАДНОФИНСКИЙ СБОРНИК, ТРУДЫ КОМИССИИ ПО ИЗУЧЕНИЮ ПЛЕМЕННОГО СОСТАВА СЕЛЕНИЯ СССР И СОПРЕДЕЛЬНЫХ СТРАН 16, ЛЕНИНГРАД: ИЗДАТЕЛЬСТВО АКАДЕМИИ НАУК СССР.

Markus, Rožanski, 2011 = МАРКУС, Е., РОЖАНСКИЙ, Ф., СОРВЕМЕННЫЙ ВОДСКИЙ ЯЗЫК. ТЕКСТЫ И ГРАММАТИЧЕСКИЙ ОЧЕРК, ТОМ I, II, САНКТ-ПЕТЕРБУРГ: НЕСТОР-ИСТОРИЯ.

Mirenkov 2002 = МИРЕНКОВ ВЛАДИМИР, «ПРОБЛЕМЫ СОХРАНЕНИЯ ИЖОРСКОГО ЯЗЫКА», Studia Slavica Finlandensia, Tomus XIX, Helsinki, 206-211.

MUSTONEN O.A.F., 1883, Muistoonpanoja Vatjan kielestä, Virittäjä I, 144-188.

Posti Lauri, SUHonen Seppo, 1964, E.N. Setälän vatjalaismuistiinpanot, Memoria Saecularis E.N.Setälä. SUST 135.

Romanova 2010 = РОМАНОВА, ЯНА, «ВОДЬ. КРАТКАЯ ИСТОРИЯ ЕЩЕ ОДНОГО ИСЧЕЗАЮЩЕГО НАРОДА», РУССКИЙ РЕПОРТЕР, 12.ОКТ 2010, 40 (168), 55-66.

TALVE Ilmari, 1981, Vatjalaista kansankulttuuria, Helsinki, Suomalais-Ugrilainen Seura.

TSVETKOv Dmitri, 2008, Vadja keele grammatika, Koostanud ja toimetanud Jüri VIIKBERG, Tallinn, Eesti Keele Sihtasutus.

TSVETKOv Dmitri, 1995, Vatjan kielen Joenperän murteen sanasto, Toim. J.LAAKSO, Lexica Societatis Fenno-ugricae XXV, Helsinki.

UKS 2004, Uurali keelte sõnastik, Tallinn, MTÜ Fenno-Ugria Asutus.

UKS 2011, Uurali keelte sõnastik, Tallinn, MTÜ Fenno-Ugria Asutus.

Vađđa kaazgad, 2003, Vađđa kaazgad, ВОДСКИЕ СКАЗКИ, ПЕРЕВОД, СОСТАВЛЕНИЕ И ОБРАБОТКА М.МУСЛИМОВ. Е.КУЗНЕЦОВА, Е.НИКОЛАЕВА, А.ГОРЕЛИКОВ, С.ЕФИМОВ, Т.ЕФИМОВА, САНКТПЕТЕРБУРГ.

$\mathrm{VE}=$ Vadja etnoloogia. Ariste käsikirjaline materjalikogu. Säilitatakse Eesti Kirjandusmuuseumi Eesti Rahvaluule Arhiivis.

VP 2005 = Paul Ariste Vadja päevikud 1942-1980, Eesti kultuuriloo allikmaterjale, 22. Koostanud, kommenteerinud ja saatesõna kirjutanud Ergo-Hart VÄSTRIK, Toimetanud ja järelsõna kirjutanud Peeter OLESK, Tartu.

\section{NOTES}

1. C'est peut-être une nationalité.

2. En russe : ЕДИНЫЙ ПЕРЕЧЕНЬ КОРЕННЫХ МАЛОЧИСЛЕННЫХ НАРОДОВ РОССИИ.

3. En russe : ПРОСТИ, ПРОЩАЙ, ЛюБИМЫЙ МОЙ.

4. cf. Bestiarium/Maaväči music and votic slide-show. Bestiarium/Maaväči votic dance music and songs from VAD`D`A album (2007) and slide-show Luutsa votic village, photos 2002-2007.

5. En estonien : Eesti Keel.

6. En estonien : Emakeele Selts.

7. En russe : СОВРЕМЕННЫЙ ВоДСКИЙ ЯЗЫК.

8. En vote: Vad'd'aa rahvaa jutud ja kaazgad. 
9. En estonien : Vadja keele sõnaraamat.

10. En vote : Vad'd'a krazgõttavõ cirja.

11. En vote: Vad'd'a paperi-lello.

12. En vote : Vunukassa baabuškassaa

\section{RÉSUMÉS}

Aujourd'hui, quatre ou cinq personnes âgées parlent vote plus ou moins couramment. Ces derniers locuteurs vivent dans trois villages à cinq ou dix kilomètres de l'embouchure de la Lauga, dans le district de Kingissepp de l'oblast' de Saint-Pétersbourg en Fédération de Russie. Jõgõperä est un ancien village construit autour d'une église ; pour des raisons administratives, Luuditsa et Liivtšülä ont été fondus en une seule unité, qui a pris le nom de Luuditsa. Dans les dix dernières années, le vote n'a servi de langue de communication dans aucune famille. Mais depuis la deuxième moitié des années 1990, les activités concernant la langue et la culture votes se sont intensifiées. C'est en 2000 que Luuditsa a fêté son $500^{\mathrm{e}}$ anniversaire, et ces festivités sont devenues très populaires dans toute l'Ingrie. En décembre 2001, Alexandr Gurinov, un artiste de Saint-Pétersbourg, a créé un drapeau vote; il existe également un blason et un hymne vote.

Les Votes n'ont jamais eu de langue écrite. Leur premier livre, Vađđa kaazgõt, a été publié en 2003-2004. L'orthographe continue à se développer. L'alphabet d'origine, de courtes traductions et des listes de vocabulaire aménagées par thèmes ont été publiés par des enthousiastes dans le journal local, qui a pour objectif premier de faire connaître la vie culturelle vote et l'histoire locale. Les activités enthousiastes de la Société de la culture vote ont conduit les Votes, le 13 octobre 2008, à être inclus dans la liste des petits peuples autochtones de la Fédération de Russie, qui énumère les peuples autochtones de Russie ayant moins de 50000 ressortissants, qui vivent sur leur territoire et suivent leur mode de vie d'origine.

Vadja keelt kõneleb enam-vähem sujuvalt kõneleb neli-viis eakat inimest. Viimased vadja keele kõnelejad elavad kolmes külas Vene Föderatsioonis Leningradi oblastis Kingissepa rajoonis Lauga jõe suudmest 5-10 kilomeetri kaugusel. Jõgõperä on vana kirikuküla, Luuditsa ja Liivtšülä on liidetud administratiivselt üheks Luuditsa külaks. Juba kümmekond aastat pole ühtegi perekonda, kes omavahel vadja keelt kõneleksid. 1990.aastate teisest poolest alates on aktiviseerunud vadja keelele ja kultuurile suunatud tegevus. 1997- 2001. aastal tegutses vadjalase Sergei Jefimovi vanemate talus vadja muuseum. Põlengu eel oli muuseumis 70 etnograafilist eset ja üle 200 vana foto. Muuseum hävis tulekahjus. 2005.aastal avati uus muuseum, mis põles 2006.aasta sügisel ja päästa õnnestus vähe. Tatjana Jefimova rajatud muuseum toimib praegu aktiivselt Internetis (watland.ru)

2000. aastal Luuditsa sai küla 500-aastaseks ja hakati tähistama küla aastapäeva. Pidu on saavutanud suure populaarsuse kõikide ingerimaalaste seas. 2001. aastal detsembris valmis Peterburi kunstnikul Aleksandr Gurinovil vadja lipp. Vadjalastel on ka vapp ja hümn.

Vadjalastel pole kunagi olnud kirjakeelt. Esimesed raamatud Vađđa kaazgõt" avaldati 2003 ja 2004. aastal. Kirjaviis on kujunemisjärgus. Vadjakeelne esialgne alfabeet, lühitõlkeid ja temaatilisi sõnaloendeid on ilmunu kohalike aktivistide poolt välja antud ajalehes „Maavätši“ , mille peaeesmärk on tutvustada vadja kultuurielu ja kodulugu. Algupäraseks võib pidada Jekaterina Kuznetsova 2010. aastal ilmunud värvimisraamatut „Vad'd'a krazgõttavõ cirja“ (Kuznetsova, Muslimov 2010). 
Vadja Kultuuriseltsi agara tegevuse tulemusel võeti vadjalased 2008.aasta 13. okt Vene Föderatsiooni väikearvuliste põlisrahvaste nimekirja (ЕДИНЫЙ ПЕРЕЧЕНЬ КОРЕННЫХ МАЛОчИслЕНныХ НАРодов России). Nimekirja kuuluvad Venemaa põlisrahvad, kelle esindajaid on vähem kui 50000 ja kes elavad oma algsel territooriumil algupärasel viisil.

Four or five elderly people speak Votian more or less fluently. The last Votian speakers live in three villages 5-10 kilometres from the mouth of the Lauga River in Kingissepp district in Leningrad Oblast in the Russian Federation. Jõgõperä is an old church village, while Luuditsa and Liivtšülä villages have been merged into a single Luuditsa village for administrative purposes. During the past ten years there has been no family whose members speak Votian among them. Activities involving the Votian language and culture have intensified since the second half of the 1990s. A Votian museum operated in a house belonging to the parents of Sergei Jefimov in the years 1997-2001. Before fire struck, the museum contained 70 ethnographic museum objects and more than 200 old photos. In 2005 a new museum was opened, but this also caught fire in the autumn of 2006 and only a few of its exhibits were saved. The museum created by Tatyana Jefimova is currently active on the Internet (watland.ru).

In 2000 the village of Luuditsa celebrated its 500th anniversary. The celebrations were very popular among all the inhabitants of Ingria. In December 2001 St. Petersburg artist Alexander Gurinov created a Votic flag. Votians also have a coat of arms and an anthem.

Votians have never had a written language. The first book, Vađđa kaazgõt, was published in 2003 and 2004. The language's orthography is still developing. The original Votian alphabet, and brief translations and lists of vocabulary arranged under various topics, have been published by enthusiasts in the local newspaper, which primarily aims to introduce Votian cultural life and local history. The colouring book Vad'd'a krazgõttavõ cirja published by Yekaterina Kuznetsova (Kuznetsova, Muslimov 2010) could be considered to be the first publication in Votian.

The ardent activities of the Votian Cultural Society led Votians to be included in the list of small indigenous peoples of the Russian Federation on October 13th 2008 (ЕДИНЫЙ ПЕРЕЧЕНЬ КОРЕННЫХ МАЛОЧИСЛЕННЫХ НАРОДОВ РОССИИ). The list includes Russia's indigenous peoples with fewer than 50000 members, and who live on their original territory in accordance with their original way of life.

\section{INDEX}

Keywords : Votian, endangered languages, Ust Luga harbour, Cultural Activities, revitalisation Mots-clés : langues en danger, port de Ust Luga, activités culturelles, revitalisation Index géographique : Alajõe, Tartu, Muuga, Riga, Tampere, Bauska, Saint-Pétersbourg, Jarvikoistšülä (БАБИно), Jõgõperä (КРАКольЕ) Joenperä (finnois) Joemperä (ingrien), Kattila (котлы), Kõrvõttula, Liivtšülä (пЕски), Luuditsa (лУжицы), Mati, Rajo (мЕжняки), Savvokkala, Soikkola, Ust-Luga, Vaipoole

Index chronologique : XXIe siècle (début), XXIe siècle

disciplines ingrien, langues fenniques, vote

nomsmotscles Estoniens, Finlandais, Ingriens, Russes, Votes 